\title{
Submodular linear programs on forests
}

\author{
Ulrich Faigle *, Walter Kern \\ Department of Applied Mathemutics. University of Twente. P.O. Box 2I7, 7500 AE Enschede. \\ The Netherlands
}

Received 7 October 1993; revised manuscript received 28 September 1995

\begin{abstract}
A general linear programming model for an order-theoretic analysis of both Edmonds' greedy algorithm for matroids and the NW-corner rule for transportation problems with Monge costs is introduced. This approach includes the model of Queyranne, Spieksma and Tardella (1993) as a special case. We solve the problem by optimal greedy algorithms for rooted forests as underlying structures. Other solvable cases are also discussed.
\end{abstract}

Keywords: Greedy algorithm; Monge property; Submodular function; Ordered set; Disributive lattice

\section{Introduction}

The idea of Edmonds [5] to interpret the matroid greedy algorithm within the primal-dual framework of linear programs derived from submodular set functions has initiated considerable interest in the investigation of combinatorial optimization problems with submodular constraints and has given rise to far-reaching generalizations of Edmonds' original model (cf., e.g., submodular functions on graphs [6], generalized polymatroids [8] or submodular systems [9]). Typically, these models are centered around totally dual integral systems of linear inequalities and thus explain many min-max relations in combinatorial optimization.

Already Hoffman [10] observed that the bipartite transportation problem becomes simpler when the cost matrix has the so-called Monge property which can be viewed as a manifestation of submodular constraints (cf. Section 2). In this case, the NW-corner

\footnotetext{
•Corresponding author. e-mail: faigle@math.utwente.nl.
} 
rule is a globally optinal algorithm, satisfying supplies and demands in a locally optimal fashion. Hoffman's result has a direct generalization to higher dimensions (cf. [1]), where the analogue of the NW-corner rule can be formulated. Bipartite graphs with Monge property have an underlying greedoid structure (cf. [4]), which might suggest greedoids as a common model for Edmond's and Hoffman's greedy algorithms. Greedoids, however, are very general and do not lend themselves easily to duality properties. On the other hand, Queyranne et al. [11] could formulate a linear programming model with submodular constraints that includes Edmonds' greedy algorithm as well as the NW-corner rule.

The present note introduces an even more general model by exhibiting the order-theoretic structure of Edmonds' and Hoffman's linear programming models. The purpose of this model is twofold: the order-theoretic approach allows us to view and to extend the results of Queyranne et al. [11] and Bein et al. [1] in a completely "coordinate-free" and conceptually much simpler context, and it naturally suggests to study the problem for more general orders.

The model in Section 2 arises from Edmonds' model essentially by restricting the constraints to the order ideals relative to some (partial) orders on the ground set and formulating the constraints only with respect to maximal elements in order ideals. In Section 3, we present a greedy algorithm that always finds a feasible dual solution if the constraints comprise all order ideals and finds a feasible dual solution, if one exists, whenever the underlying order is a rooted forest. Optimality of the algorithm will follow if we can exhibit an associated primally feasible solution.

Primal feasibility is addressed in Section 4, where we give a positive answer in the case of submodular constraints and a rooted forest as underlying order structure. It turns out that our algorithm works in the same way for arbitrary orders if the constraint function is not only submodular but also monotone. In general, however, it is not even clear whether submodularity is the right notion for the analysis of the greedy algorithm.

\section{The linear programming model}

Let $P=(E, \leqslant)$ be a (partial) order on the finite set $E$, and let $\mathscr{A}$ be a arbitrary (but fixed) family of subsets of $E$.

For each $A \in \mathscr{A}$, we denote by $A^{+}$the collection of maximal elements of $A$ relative to the order induced by $P$ on $\mathscr{A}$.

Consider any function $f: \mathscr{A} \rightarrow \mathbb{R}$ together with a weighting $c: E \rightarrow \mathbb{R}$. We are interested in the linear program

$$
\begin{array}{ll}
\max & \sum_{e \in E} c_{e} x_{e} \\
\text { s.t. } & \sum_{e \in A} x_{e} \leqslant f(A), \text { for all } A \in \mathscr{A},
\end{array}
$$


and its dual

$$
\begin{aligned}
& \min \sum_{A \in \mathscr{A}} f(A) y_{A} \\
& \text { s.t. } \sum_{\substack{A \in \mathcal{N}^{\prime} \\
e \in A^{+}}} y_{A}=c_{e}, \quad \text { for all } e \in E \text {, } \\
& y_{A} \geqslant 0, \quad \text { for all } A \in \mathscr{A} \text {. }
\end{aligned}
$$

It is important to clarify the role of the subset family $\mathscr{A}$ vs the order relation $P$ in our model. $\mathscr{A}$ itself is independent of $P . P$, however, determines which elements are "maximal" in the members of $\mathscr{A}$. Thus, considering the same family $\mathscr{A}$ relative to a different order relation $Q$ will lead to different constraints in the linear programs (1) and (2) and hence to different optimization problems.

We note some special cases of our model. If $P$ is the trivial order on $E$ and $\mathscr{A}$ comprises all subsets of $E$, the feasible solutions of (1) form a submodular system in the sense of Fujishige [9] provided $f$ is submodular, i.e.

$$
f(A \cup B)+f(A \cap B) \leqslant f(A)+f(B), \text { for all } A, B \in \mathscr{A} .
$$

The nonnegative vectors of a submodular system constitute a polymatroid as introduced by Edmonds [5]. The vectors $x$ in a submodular system attaining the equality

$$
\sum_{e \in E^{+}} x_{e}=f(E)
$$

make up the associated base polytope. The generalized polymatroids of Frank and Tardos [8] are projections of submodular base polytopes along one coordinate. All these structures generalize matroids on one hand, but allow, on the other hand, a structural analysis within the framework of classical matroid theory (cf. [7]).

Let us assume now that each $\mathscr{A} \in \mathscr{A}$ is an (order) ideal relative to $P$, i.e.,

$$
e \leqslant a \text { implies } e \in A, \text { for all } a \in A, e \in E \text {. }
$$

Each ideal $A$ is uniquely determined by its set $A^{+}$of maximal elements. In the case where $P$ is the union of $k$ pairwise unrelated chains, each $A \in \mathscr{A}$ may thus be though of as a $k$-dimensional vector, whose components indicate the respective maximal elements of $A$ in each chain. When is closed under union and intersection and $f$ is submodular, we obtain the model of Queyranne et al. [11].

If $P$ consists of $k=2$ unrelated chains, each ideal $A$ with $\left|A^{+}\right|=2$ can be interpreted as a "line" of the bipartite graph whose two color classes are the respective chains. So (2) is a transportation problem with the $c_{e}$ 's as "demands" and "supplies" respectively and "costs" $f(A)$ on the lines. Submodularity of $f$ in this context is tantamount to saying that the costs of our transportation problem have the so-called Monge property. (For a recent extensive survey on Monge optimization see, e.g. [3].)

The work of Hoffman [10] in particular implies that the so-called NW-corner rule solves a transportation problem if the cost matrix has the Monge property. The $\mathrm{NW}$-corner rule iterates the following step: try to satisfy as much of the demand (or 
supply) with the currently lexicographically largest line and then remove it from the bipartite graph.

Bein et al. [1] observed that the NW-comer rule solves also the general $k$-dimensional transportation problem on the complete $k$-partite graph if the costs are submodular. Queyranne et al. [11] investigate it within the primal-dual setting of linear programming, stipulating only that the lines of the (not necessarily complete) $k$-partite graph correspond to a family $\omega^{\circ}$ of ideals that is closed under union and intersection.

Returning to the general problem (1) and (2) respectively, let $\mathscr{L} \subseteq \mathscr{A}$ be a subfamily of $\mathscr{A}$. We associate with $\mathscr{L}$ the incidence matrix $L$ whose rows are indexed by the sets $A$ in $\mathscr{L}$ and columns by the elements $e \in E$ such that

$$
L_{A, \ell^{\prime}}= \begin{cases}1, & \text { if } e \in A^{+} \\ 0, & \text { otherwise. }\end{cases}
$$

Denoting by $f \mid z$ the restriction of $f$ to $\mathscr{f}$, we say that the vector $x \in \mathbb{R}^{E}$ is primally $\mathscr{Z}$-greedy if

$$
L x=\left.f\right|_{2}
$$

or, equivalently.

$$
\sum_{e \in A^{-}} x_{c}=f(A) \text { for all } A \in \mathscr{L} \text {. }
$$

Similarly. we call a vector $y=\left(y_{4} \mid A \in \mathscr{L}\right)$ dually $\mathscr{L}$-greedy if

$$
y^{\top} L=c^{\mathrm{T}} \text { and } y \geqslant 0 \text {. }
$$

Observe that any dually $\mathscr{L}$-greedy $y$ extends to a feasible solution $\bar{y}$ for $(2)$ in a natural way by setting

$$
\bar{y}_{A}= \begin{cases}y_{A}, & \text { if } A \in \mathscr{E}, \\ 0, & \text { otherwise. }\end{cases}
$$

A primally $\mathscr{E}$-greedy vector $x$ will generally not be feasible for (1). We note, however, the following.

Lemma 2.1. Let $x$ be primally and $y$ dually Lreedy. Then,

$$
\sum_{e \in E} c_{e} x_{c}=\sum_{A \in \mathscr{Z}} f(A) \bar{y}_{A} \text {. }
$$

In view of Lemma 2.1, the problems (1) and (2) are solved optimally once we exhibit some family $\mathscr{L} \subseteq \mathscr{W}$ that gives rise to feasible $\mathscr{L}$-greedy solutions $x$ and $y$.

\section{The greedy algorithm}

Having the optimal solvability of the primal-dual pair (1) and (2) of problems in mind, we can make the following assumptions.

$($ A.0) $\emptyset \in \mathscr{A}$ and $f(\emptyset)=0$. 
$\left(f(\emptyset)<0\right.$ would make (1) infeasible while $f(\emptyset)>0$ would imply $y_{0}=0$ in any optimal solution $y$ of (2).)

(A.1) every $A \in \mathscr{A}$ is uniquely determined by its set $A^{+}$of maximal elements.

Because of (A.1), there is no loss of generality when we henceforth assume that each $A \in \mathscr{A}$ is an order ideal relative to $P$.

(A.2) For every $e \in E$, there exists some $A \in \mathscr{A}$ such that $e \in A^{+}$.

Our next assumption, however, imposes a proper restriction on the general model of Section 2 by requiring that $\mathscr{A}$ be closed under taking unions and intersections.

(A.3) For every $\mathrm{A}, \mathrm{B} \in \mathscr{A}, A \cup B, A \cap B \in \not{A}$.

We will from now on always assume the properties (A.0)-(A.3) to hold. In the algorithm below, we deal with the following quantities iteratively:

(i) $c_{\min }=\min \left\{c(e) \mid e \in E^{+}\right\}$,

(ii) $T_{\text {min }}$ is some member of $\mathscr{A}$ that is maximal (with respect to set-theoretic containment) such that there exists some $e \in E^{+} \backslash T_{\min }$ with $c(e)=c_{\min }$.

Note that $T_{\text {min }}$ exists (because $\emptyset \in \mathscr{*}$ ) as long as $E \neq \emptyset$. However, $T_{\text {mil }}$ is generally not unique. For our purposes, just some $T_{\text {nin }}$ with property (ii) suffices.

Lemma 3.1. Let the problem (2) be feasible. Then,

(a) $c(e) \geqslant 0$, for all $e \in E$;

(b) $c(e)=c_{\min }$, for all $e \in E^{+} \backslash T_{\min }$;

(c) for all $e, f \in E \backslash T_{\text {min }}$ and $A \in \mathscr{A}, e \in A^{+} \Leftrightarrow f \in A^{+}$;

(d) $E \backslash T_{\min } \subseteq E^{+}$.

Proof. Let $y \in \mathbb{R}^{\leftrightarrow}$ be feasible for (2). Then statement (a) is implied by the inequality

$$
0 \leqslant \sum_{\substack{A \in \mathcal{H}^{+} \\ e \in A^{+}}} y_{A}=c_{c} .
$$

Consider now $e, f \in E^{+} \backslash T_{\min }$ such that $c(e)=c_{\min }$. Then each $A \in \mathscr{A}$ with $f \in A^{+}$ must also satisfy $e \in A^{+}$(otherwise $T_{\min } \cup A \in \mathscr{A}$ would strictly contain $T_{\text {min }}$ - in contradiction to the choice of $T_{\min }$ ). So we have

$$
c(f)=\sum_{\substack{A \in \mathcal{A}^{\prime} \\ f \in A^{\prime}}} y_{a} \leqslant \sum_{\substack{A \in \mathcal{N}^{-} \\ e \in A^{-}}} y_{A}=c(e)=c_{\min } .
$$

This proves (b) and one direction of the equivalence claimed in (c). The other direction follows in the same way because $c(e)=c(f)=c_{\text {min }}$.

To see (d), suppose there exists some $e \in E \backslash T_{\min }$ which is not a maximal element of $E$. By assumption (A.2), we know $e \in A^{+}$for some $A \in \mathscr{A}$. Because $T_{\min }$ is an ideal, there is some $f \in E^{+} \backslash T_{\min }$ such that $e<f$. Now $e \in A^{+}$implies $f \notin A$. So $f \notin T_{\min } \cup$ $A$ and $c(f)=c_{\min }$ contradict the choice of $T_{\text {min }}$. 
The analysis of Lemma 3.1 suggests the following heuristic for finding a feasible solution for (2). Thereby we use the notation

$$
\mathscr{\infty}(T)=\{A \in \mathscr{\infty} \mid A \subseteq T\} \text {. }
$$

\section{Greedy Algorithm}

$$
\begin{aligned}
& \text { Initialize: } y_{A} \leftarrow 0 \text {, for all } A \in \mathscr{A} \text { : } \\
& \text { Iterate:WHILE } E \neq \emptyset D O \text { : } \\
& \text { determine } c_{\min } \text { and some } T_{\min } \text { : } \\
& y_{E} \leftarrow c_{\min } \\
& c(e) \leftarrow\left[c(e)-c_{\text {min }}\right] \text {, for all } e \in E^{+} \text {; } \\
& \mathscr{A} \leftarrow \mathscr{A}\left(T_{\text {nin }}\right) \text {; } \\
& E \leftarrow T_{\min } \square \text {. }
\end{aligned}
$$

Theorem 3.1. Assume that consists of all order ideals relative to $P$. Then the Greedy Algorithm produces a feasible solution for (2).

Proof. If A contains all ideals relative to $P$, then $T_{\min }=E \backslash\{e\}$ in each iteration of the Greedy Algorithm. Hence it is easy to see by induction on the size of the ground set $E$ that the greedy solution is dually feasible.

If $\mathscr{A}$ does not contain all ideals, it is easy to see that (1) may be unbounded, i.e., (2) may be infeasible. Generalizing a result of Queyranne et al. [11], we now want to exhibit a class of orders $P$ where the Greedy Algorithm detects infeasibility.

Let $y=\left(y_{A} \mid A \in \mathscr{A}\right)$ be a feasible vector for (2) and consider $A, B \in \mathscr{A}$ such that

$$
A \neq A \cup B \neq B \text {. }
$$

We define the vector $y^{\prime}=\operatorname{swap}(y ; A, B)$ via

$$
y^{\prime}(S)= \begin{cases}y(S)+\lambda, & \text { if } S=A \cup B \text { or } S=A \cap B, \\ y(S)-\lambda, & \text { if } S=A \text { or } S=B, \\ y(S), & \text { otherwise. }\end{cases}
$$

where $\lambda=\min \left(y_{A}, y_{B}\right)$.

Lemma 3.2. Let the order $P$ be such that each element $e \in E$ has at most one upper neighbor relative to $P$. Then also the vector $y^{\prime}=\operatorname{swap}(y ; A, B)$ is feasible for (2).

Proof. Because $\lambda=\min \left(y_{A}, y_{B}\right), y^{\prime}$ is nonnegative. To check that also the other feasibility conditions of (2) are satisfied for each $e \in E$, we distinguish three cases.

(1) $e \in A^{+}$and $e \in B^{+}$. Then $e \in(A \cup B)^{+}$and $e \in(A \cap B)^{+}$and no violation can incur.

(2) $e \in A^{+}$and $e \notin B^{+}$. There are two subcases. If $e \in B$, then $e \notin(A \cup B)^{+}$but $e \in(A \cap B)^{+}$. If $e \notin B$, then $e \in(A \cup B)^{+}$and $e \notin(A \cap B)^{+}$. So in either case feasibility remains to hold. 
(3) $e \notin A^{+}$and $e \notin B^{+}$. Then e $\notin(A \cup B)^{+}$. Moreover, also $e \notin(A \cap B)^{+}$must hold. This is so because $e$ has an upper neighbor $f$, say, in $A$ and some upper neighbor $f^{\prime}$ in $B$. By our assumption on $P$, we know $f=f^{\prime}$ and therefore $f \in(A \cap B)$ shows that $e$ cannot be maximal in $A \cap B$.

Theorem 3.2. Let the order $P$ be such that each element $e \in E$ has at most one upper neighbor relative to $P$. Then the Greedy Algorithm constructs a feasible solution for (2) if and only if (2) is feasible.

Proof. Assume that (2) is feasible. In view of the iterations in the Greedy Algorithm, it suffices to show that (2) admits a feasible solution $y$ with $y_{E}=c_{\min }$. The feasibility of the greedy solution then easily follows by induction on the number of iterations.

Choose $y$ so that $y_{E}$ is as large as possible. Suppose $y_{E}<c_{\min }$. Because $y$ is feasible, for each $e \in E^{+}$there must exist some $A \in \mathscr{A} \backslash\{E\}$ with $e \in A$ and $y_{A}>0$. So we can find a minimal $k$ and ideals $A_{1}, \ldots, A_{k} \in \mathscr{A} \backslash\{E\}$ such that $y_{A i}>0$ for $i=1, \ldots, k$, and

$$
A_{1} \cup A_{2} \cup \cdots \cup A_{k}=E \text {. }
$$

We now carry out $k$ swaps as follows. Set $y^{(1)}=y$ and $Z^{(1)}=A_{1}$. For $i=2, \ldots, k$, set

$$
y^{(i)}=\operatorname{swap}\left(y^{(i-1)} ; Z^{(i-1)}, A_{j}\right) \quad \text { and } \quad Z^{(i)}=Z^{(i-1)} \cup A_{i}
$$

By Lemma 3.2, $y^{(k)}$ is feasible. Moreover, the swap operation implies for $i=2, \ldots, k$,

$$
y_{Z(i)}^{(i)}>y_{Z(i)}^{(i-1)} .
$$

Noting $Z^{(k)}=E$, we therefore conclude $y_{E}^{(k)}>y_{E}$, which contradicts the choice of $y$.

We will say that the order $P$ is a rooted forest if $P$ satisfies the hypothesis of Theorem 3.2. Since the linear program (1) is always feasible, Theorem 3.2 can be interpreted as saying that, in the case of rooted forests, both (1) and (2) have optimal solutions if and only if the Greedy Algorithm produces a feasible solution. We will discuss the question of the optimality of the greedy solution in the next section.

Let $\mathscr{L}$ be the collection of those $A \in \mathscr{A}$ that occur as some " $E$ " during the execution of the Greedy Algorithm. Then $\mathscr{L}$ is a monotone chain

$$
\emptyset=A_{0} \subset A_{1} \subset \cdots \subset A_{k} \subset A_{k+1}=E .
$$

In the case of the $d$-dimensional transportation problem, the sequence $\left(A_{k+1}^{+}, A_{k}^{+}, \ldots\right.$, $A_{1}^{+}$) corresponds to the sequence of $d$-dimensional lines that are processed by an application of the NW comer rule.

In the model of Edmonds [5], $\mathscr{A}$ comprises all subsets of $E$. So the Greedy Algorithm implicitly orders $E=\left\{e_{1}, \ldots, e_{n}\right\}$ according to

$$
c\left(e_{1}\right) \geqslant c\left(e_{2}\right) \geqslant \cdots \geqslant\left(e_{n}\right)
$$


and obtains $\mathscr{F}$ to be the collection of subsets of the form

$$
E_{i}=\left\{e_{1}, c_{2}, \ldots, e_{i}\right\}, \quad i=1, \ldots, n .
$$

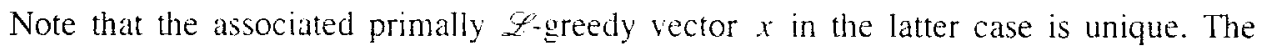
iterative solution of the linear equality system (6) is usually referred to as "Edmonds" greedy algorithm" and generalizes the matroid greedy algorithm.

As pointed out in Section 2, the crucial point in our approach is the question whether there exists a primally $\mathscr{L}$-greedy solution that is also feasible for (1). We address this question in the next section.

\section{Primal feasibility}

We assume throughout this section that the optimization problem (2) is feasible and that the chain

$$
\mathscr{Z}=\left\{\emptyset=A_{0} \subset A_{1} \subset \cdots \subset A_{h+1}=E\right\}
$$

of members of $\mathscr{A}$ is generated by a run of the Greedy Algorithm in Section 3.

In particular, we require the properties (A.O)-(A.3) to be satisfied by $\mathscr{A}$.

Under all these assumptions, we consider the primally $\mathscr{L}$-greedy vector $x \in \mathbb{R}^{E}$, i.e., a vector satisfying

$$
\sum_{e \in A_{i}} x_{i}=f\left(A_{l}\right), \text { for } l=1.2 \ldots, k, k+1
$$

Recall that $f: \mathscr{A} \rightarrow \mathbb{R}$ is submodular if for all $A, B \in \mathscr{A}$,

$$
f(A \cup B)+f(A \cap B) \leqslant f(A)+f(B) .
$$

Moreover, recall that $P=(E, \leqslant)$ is a rooted forest if each element of $E$ has at most one upper neighbor relative to the order $P$.

Theorem 4.1. Let $P$ be a rooted forest and $f: \mathscr{R} \rightarrow \mathbb{R}$ be submodular. Then the

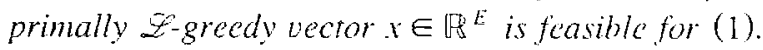

Proof. We argue by induction on $|E|$. Recalling that $A_{k}=T_{\text {min }}$, we may thus assume that the restriction of $x$ to $A_{k}$ is feasible with respect to the accordingly restricted problem (1). In other words, we may assume

$$
\sum_{e \in A^{+}} x_{t} \leqslant f(A), \text { for all } A \in \mathscr{O} \text { with } A \subseteq A_{k} .
$$

We now distinguish two cases. If $\left|E \backslash A_{k}\right| \geqslant 2$, property (c) in Lemma 3.1 allows us to identify the elements of $E \backslash A_{k}$ into one single element so that essentially the same problem arises on a strictly smaller ground set. Hence we conclude by induction that $x$ is feasible for (1). 
It remains to settle the case $E \backslash A_{k}=\{g\}$. say. We must show that

$$
\sum_{e \in A^{+}} x_{e} \leqslant f(A)
$$

holds for all $A \in \mathscr{A}$ with $g \in A$. The submodularity of $f$ now yields with $B=A \backslash\{g\}$ $=A \cap A_{k}$ :

$$
f(A) \geqslant f\left(A \cap A_{k}\right)+f\left(A \cup A_{k}\right)-f\left(A_{k}\right)=f(B)+f(E)-f\left(A_{k}\right) .
$$

Because $B$ is contained in $A_{k}$, we know by induction that

$$
f(B) \geqslant \sum_{c \in B^{+}} x_{\epsilon^{\prime}} .
$$

Moreover, since $x$ is $\mathscr{L}$-greedy,

$$
f(E)-f\left(A_{k}\right)=x_{k}-\sum_{\substack{c \in A_{k}^{+} \\ c<k}} x_{c} .
$$

Because $P$ is a rooted forest, we have the equality

$$
\sum_{\substack{e \in A_{k}^{+} \\ e<g}} x_{e}=\sum_{\substack{e \in B^{+} \\ e<g}} x_{e} .
$$

Hence,

$$
f(B)+f(E)-f\left(A_{k}\right) \geqslant \sum_{e \in A^{\prime}} x_{e^{\prime}}
$$

which yields the desired inequality.

Recall that the Greedy Algorithm of Section 3 constructs an integral feasible solution if (2) is feasible and the weighting $c: E \rightarrow \mathbb{R}$ is integral. So we may state the following.

Corollary 4.1. Let $P$ be a rooted forest and $f: \mathscr{A} \rightarrow \mathbb{R}$ submodular. Then,

$$
\sum_{c \in A^{+}} x_{e} \leqslant f(A), \quad \text { for all } A \in \mathscr{A},
$$

is a totally dual integral system of linear inequalities.

Theorem 4.1 admits a certain converse.

Proposition 4.1. Let $P$ be a rooted forest and $f: \mathscr{G} \rightarrow \mathbb{R}$ be such that there exists some feasible primally $\mathscr{L}$-greedy vector for (1) whenever $\mathscr{L}$ arises from the Greedy Algorithm. Then $f$ is submodular.

Proof: It is a well-known fact and easy to prove by induction that $f$ is submodular if $f(A \cup S)+f(A \cup T) \geqslant f(A \cup S \cup T)+f(A)$

holds for every $A \in \mathscr{A}$ and subsets $S$ and $T$ such that $A \cup S \cup T$ is an upper neighbor of both $A \cup S$ and $A \cup T$ in the lattice $\mathscr{A}$. (Observe that in this case $A$ is a lower neighbor 
of both $A \cup S$ and $A \cup T$ in $\mathscr{A}$ (for more details on (distributive) lattices, see, e.g., [2]).)

Arguing again by induction on $|E|$, we may assume that $A \cup S \cup T=E$.

Choosing the weighting $c$ appropriately, it is not difficult to see that the greedy algorithm may generate some

$$
\mathscr{Z}=\left\{\emptyset \subset A_{1} \subset \cdots \subset A \subset(A \cup S) \subset E\right\} .
$$

In view of property (c) in Lemma 3.1, we may assume $|E \backslash(A \cup S)|=1$ and, similarly. $|E \backslash(A \cup T)|=1$. So $A \cup S=A \cup\{s\}$ and $A \cup T=A \cup\{t\}$ for some $s, t \in E$.

Consicler now the primally feasible $\mathscr{L}$-greedy vector $x$. Then,

$$
\begin{aligned}
& f(A)=\sum_{\epsilon \in A^{*}} x_{*} \\
& f(A \cup S)=\sum_{e \in A} x_{i}-\sum_{\substack{e \in A^{\prime} \\
e^{\prime}<i}} x_{i}+x_{s} .
\end{aligned}
$$

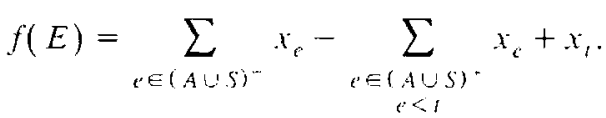

Since $P$ is a rooted forest, the lower neighbors of $s$ and $t$ form two disjoint subsets. From the feasibility of $x$ we can, therefore, conclude

$$
\begin{aligned}
f(A \cup T) & \geqslant \sum_{e \in(A \cup T)^{-}} x_{e} \\
& =\sum_{e \in A^{-}} x_{e}-\sum_{\substack{c \in A^{-} \\
e<1}} x_{c}+x_{i} \\
& =f(E)+f(A)-f(A \cup S) .
\end{aligned}
$$

Moreover, the analogue of Theorem 4.1 may be false if $P$ is not a rooted forest.

Example 4.1. Consider the order $P$ on $E=\{a, b, c, d, e\}$ with nontrivial relations $a<c, b<d$ and $b<e$. We let $\&$ consist of all order ideals $A$ and set

$$
f(A)= \begin{cases}1, & \text { if } A=\{a, c\}, \\ 0, & \text { otherwise }\end{cases}
$$

One easily verifies that $f$ as submodular and $x=(0,-1,1,-1,0)$ is the unique primally $\mathscr{E}$-greedy vector with respect to the weighting

$$
c_{a}=4, \quad c_{h}=2, \quad c_{c}=4, \quad c_{d}=1, \quad c_{c}=0 .
$$

For $A=\{a, b, c, e\}$, we now have

$$
1=x_{c}+x_{c}=\sum_{i \in A^{\prime}} x_{i}>0=f(A) \text {. }
$$

i.e., $x$ is not feasible.

In spite of Example 4.1. rooted forests may not be necessary for the Greedy Algorithm to perform optimally if the function $f$ behaves nicely with respect to the dual solution. 
Theorem 4.2. Let the order $P$ be arbitrary and $f: \mathscr{A} \rightarrow \mathbb{R}$ be submodular. Let furthermore the chain $\mathscr{L}$ be obtained by the Greedy Algorithm. Then every nonnegative primally $\mathscr{L}$-greedy vector $x$ is feasible for (1).

Proof. We may argue as in the proof of Theorem 4.1, remarking that the inequality

$$
\sum_{\substack{e \in A^{+} \\ e<g}} x_{e} \leqslant \sum_{\substack{e \in B^{+} \\ e<g}} x_{e}
$$

must hold if the $x_{e}$ 's are nonnegative.

Corollary 4.2. If $f$ is monotone on the chain $\mathscr{L}$, then there exists a feasible primally $\mathscr{L}$-greedy vector.

We must leave it as an open problem how to analyze greedy algorithms for the submodular programs (1) and (2) when $P$ is not a rooted forest or when Theorem 4.2 does not apply.

It is also not clear whether submodularity of $f$ is the right notion for the greedy algorithm when $P$ is not a rooted forest. For example, there exist orders $P$ and nonsubmodular functions $f$ such that every greedy vector is nonnegative and feasible.

Example 4.2. Consider the order $P$ on $E=\{a, b, c\}$ given by the nontrivial relations $a<b$ and $a<c$. Define $f$ on the lattice $\mathscr{A}$ of all ideals by

$$
f(a)=f(E)=2, \quad f(a, b)=f(a, c)=1 .
$$

Then $f$ is not submodular but the - in this example unique - greedy vector is nonnegative and feasible.

\section{Acknowledgements}

We thank M. Queyranne for interesting discussions on the present model and for pointing out an error in an earlier version of Example 4.1 and U. Krüger for his careful reading of the manuscript.

\section{References}

[1] W.W. Bein, P. Brucker, J.K. Park and P.K. Pathak, "A Monge property for the $d$-dimensional transportation problem," Discrete Applied Mathematics 58 (1995) 97-109.

[2] G. Birkhoff, Latice Theory. American Mathematical Society Colloquium Publications, Vol. 25 (American Mathematical Society, Providence, RI, 1967).

[3] R.E. Burkard, B. Klinz and R. Rudolf, "Perspectives of Monge properties in optimization," Discrete Applied Mathematics. to appear.

[4] U. Derigs, O. Goecke and R. Schrader. "Monge sequences and a simple assignment algorithm," Discrete Applied Mathematics 15 (1986) 241-248. 
[5] J. Edmonds, "Submodular functions, matroids and certain polyhedra," in: R. Guy et al., eds., Combinatorial Structures and their Applications (Gordon and Breach, New York, 1970) pp. 69-87.

[6] J. Edmonds and R. Giles, "A min-max relation for submodular functions on graphs," in: P.L. Hammer, E.L. Johnson. B.H. Korte and G.L. Nemhauser. eds., Studies in /meger Programming, Annals of Discrete Mathematics, Vol. 1 (North-Holland. Amsterdam, 1977) pp. 185-204.

[7] U. Faigle, "Matroids in combinatorial optimization." in: N. White, ed., Combinutorial Geometries, Encyclopedia of Mathematics and its Applications. Vol. 29 (Cambridge University Press, Cambridge. 1987) pp. 161-210.

[8] A. Frank and E. Tardos. "Generalized polymatroids and submodular flows," Mathematical Programming $42(1988) 489-563$.

[9] S. Fujishige. Submotular Functions: and Oprimization. Annals of Discrele Mathematics, Vol. 47 (North-Holland. Amsterdam, 1990).

[10] A.J. Hoffman. "On simple linear programming problems," in: V. Klee, ed., Convexity, Proceedings of Symposia in Pure Mathematics. Vol. 7 (American Mathematical Society, Providence, RI, 1963) pp. $317-327$.

[11] M. Queyranne. F. Spicksma and F. Tardella. "A general class of greedily solvable linear programs," in: U. Faigle and C. Hoede. eds. Srd Tisente Workshop on Graphs and Combinatorial Optimization, Memorandum No. 1132 (Department of Applied Mathematics. University of Twente, Enschede. 1993). 